\title{
Single Photon LiDAR Technology for Gas Imaging
}

\author{
Peter Droegmoeller ${ }^{1}$, Murray Reed ${ }^{2}$ \\ ${ }^{1}$ AMETEK Land, Stubley Lane, Dronfield S18 1DJ, UK, \\ ${ }^{2}$ QLM, Unit DX, Albert Road, St Philips, Bristol, BS2 OXJ, UK \\ peter.droegmoeller@ametek.com
}

\begin{abstract}
Summary:
A LiDAR camera system combined with a laser tuned across a specific gas absorption can measure the distance and the quantity of gas present in the surrounding area. New technology now enables this measurement to be taken without the use of large expensive lasers.
\end{abstract}

Keywords: LiDAR, rapidly tunable laser, gas imaging, Single Photon Avalanche Diodes, SPADS

\section{Background, Motivation an Objective}

Monitoring greenhouse gases in becoming a focus in the effort to reduce global warming. Gases can be detected by measuring the specific absorption bands, e.g. methane absorbs at $1651 \mathrm{~nm}$. To monitor gases with existing technology infrared cameras tuned to the specific absorption band are used. To be visible the gas needs to be either at a different temperature, e.g. colder due to a release from high pressure vessel or illuminated by a broad band head source like the sun. Alternatively, the illumination can be done with a wavelength specific laser, where new developments in the field of single photon avalanche diode (SPADS) will allow the use of much smaller lasers. The goal was to build a camera system using LiDAR technology for gas imaging and quantification. LiDAR stands for Light Detection And Ranging and is already more commonly adopted in the automotive industry on autonomous vehicles and driver support systems. The range of these systems are limited to $30-40 \mathrm{~m}$ when high powered NIR lasers $(905 \mathrm{~nm})$ are used. The range can be extended to $200 \mathrm{~m}$ by using SWIR lasers at $1550 \mathrm{~nm}[1]$.

\section{Description of the New Method or System}

The active imager consists of a rapidly tunable distributed feedback (DFB) laser diode, scanning optics, a single photon detector and electronics for signal processing. The laser is sending out at various wavelength close to the desired absorption band of the to be measured gas. The signal will scatter on solid objects and parts of the signal received back into the camera. If the gas was present in the sight path, the gas specific absorption will be visible in the spectral return signal. For methane, the strong absorption band at $1651 \mathrm{~nm}$ can be used.

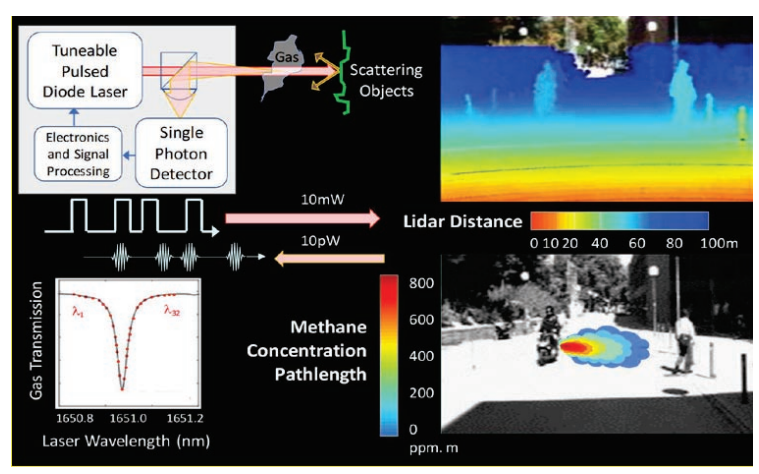

Fig. 1. System diagram of Single-Photon Diode Laser Gas Imaging LiDAR

The main challenge besides the fast tuning of the laser diode is the small return signal from the objects in the range of $\mathrm{pW}$. It has been overcome with the use of uncooled InGaAs SWIR Single Photon Avalanche Diodes, which are commonly used in telecommunication fibre optics. The absorption band of methane matches the spectral response of the detector (1000 to $1700 \mathrm{~nm})$.

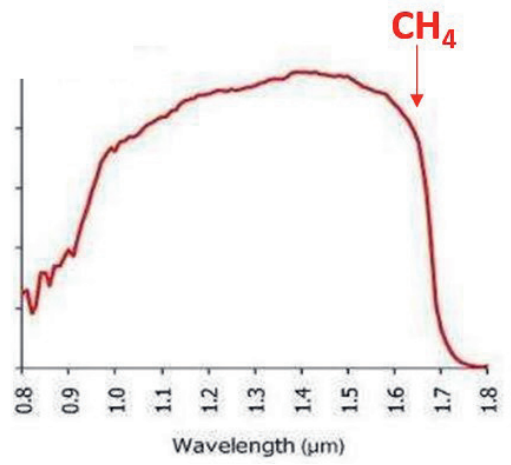

Fig. 2. Spectral response of InGaAs SWIR Single-Photon Diode 


\section{Results}

The sensitivity of the novel SWIR SPAD enabled the use of a low power semiconductor laser. The laser beams through the gas and reflects off the surroundings, whereas the methane concentration levels detected were in the PPM range even at long distances above $100 \mathrm{~m}$.

A highspeed scanning system was used to generate a $2 \mathrm{D}$ image of the gas concentration with was then overlayed onto the visible camera image. The result in Fig 3 are taken at 100m distance with the full image scan completed within 1 minute.
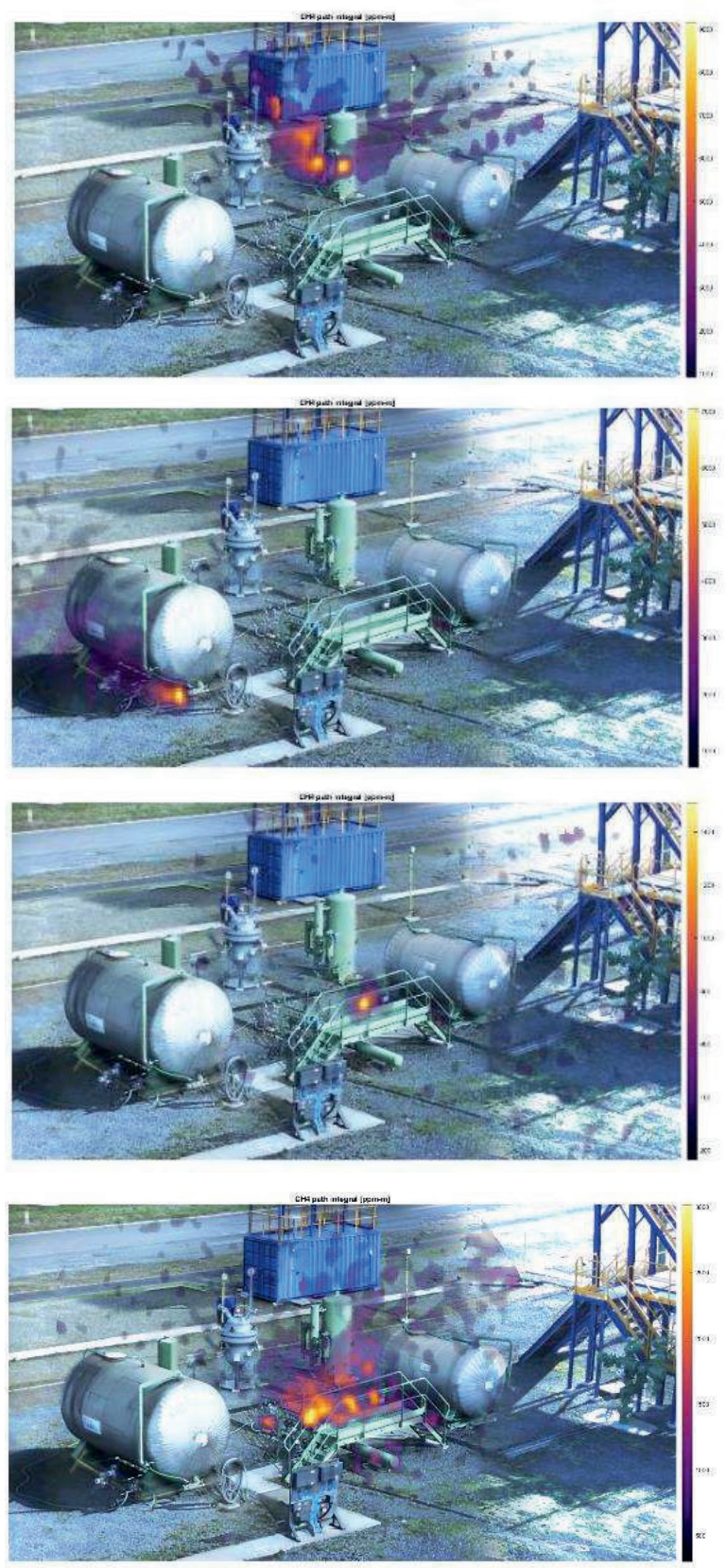

Fig. 3. Methane leak detection at test site
Blind leak simulation trial have confirmed a good correlation between the measured leak rate and the actual leak rate (Fig 3 ).

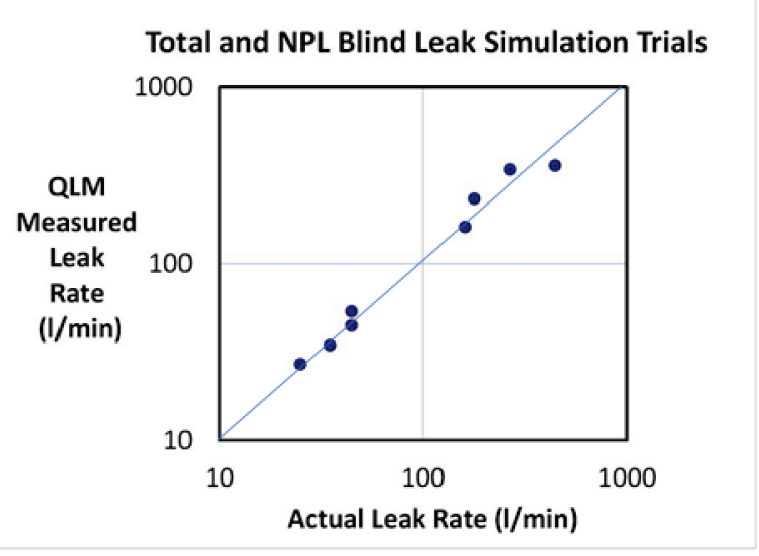

Fig. 4. Methane leak detection at test site

The availability of new laser and SPAD wavelength will allow the detection of other gases besides methane. Possible candidates for SWIR based detections are $\mathrm{CO}$ and $\mathrm{CO}_{2}$. An expansion into the MWIR spectrum will be of particular interest to the petrochemical industry for the qualification and quantification of process gases.

\section{Aknowledgement}

The results are based on research funded by the UK national quantum technologies programme as part of the SPLICE project.

\section{References}

[1] Corneliu Rablau, Lidar: a new self-driving vehicle for introducing optics to broader engineering and non-engineering audiences, Proceedings Volume 11143, 15th Conference on Education and Training in Optics and Photonics; Quebec, 2019

[2] $\mathrm{Ai}, \mathrm{Xiao}$, et al. "Pseudo-random single photon counting for space-borne atmospheric sensing applications." Aerospace Conference, 2014 IEEE. IEEE, 2014.

[3] BRITESPACE consortium, "BRITESPACE High Brightness Semiconductor Laser Sources for Space Applications in Earth Observation FINAL PUBLISHABLE REPORT", 2016 